\title{
Numerical analysis of fluid flow properties in a partially perforated horizontal wellbore
}

\author{
Mohammed Abdulwahhab Abdulwahid ${ }^{1, ~ *, ~ S a d o u n ~ F a h a d ~ D a k h i l ~}{ }^{2}$, I. N. Niranjan Kumar ${ }^{3}$ \\ ${ }^{1}$ Marine Engineering Department, Andhra University, Visakhapatnam, India \\ ${ }^{2}$ Fuel \& Energy Department, Basrah Technical College, Basrah, Iraq \\ ${ }^{3}$ Marine Engineering Department, Andhra University, Visakhapatnam, India \\ Email address: \\ Mohw2002@yahoo.com (M. A. Abdulwahid), drsadoun2@gmail.com (S. F. Dakhil), neeru9@yahoo.com (I. N. N. Kumar)
}

\section{To cite this article:}

Mohammed Abdulwahhab Abdulwahid, Sadoun Fahad Dakhil, I. N. Niranjan Kumar. Numerical Analysis of Fluid Flow Properties in a Partially Perforated Horizontal Wellbore. American Journal of Energy Engineering. Vol. 2, No. 6, 2014, pp. 133-140.

doi: $10.11648 /$ j.ajee.20140206.12

\begin{abstract}
The pressure drops in horizontal wellbores, acceleration, wall friction, perforation roughness, and fluid mixing are analyzed in a partially perforated wellbore. It was demonstrated that the perforation inflow actually reduced the total pressure drop. The pressure drop due to perforation roughness was eliminated by the perforation inflow when the ratio of radial perforation flow to axial pipe flow rate reached a certain limit. Three dimensional numerical simulations on a partially perforated pipe with 150 perforations, geometrically similar with wellbore casing (12 SPF, and 60 phasing) were presented and analyzed. Numerical simulations by commercial code CFX were also conducted with Reynolds numbers ranging from 28,773 to 90,153 and influx flow rate ranging from 0 to $899 \mathrm{lit} / \mathrm{hr}$ to observe the flow through perforated pipe, measure pressure drops, friction factors and pressure loss coefficients. The acceleration pressure drop might be important compared with the frictional pressure drop. The numerically calculated results using $k-\varepsilon$ model were compared with the experimental results. The numerical solutions agreed well with the experimental data.
\end{abstract}

Keywords: Pressure Drop, Perforation, Numerical, Radial Flow, Wellbore

\section{Introduction}

Over the last decade, flows through horizontal wells have become a well-established technology for the recovery of oil and gas. A considerable amount of analytical and experimental work has been published on various aspects of horizontal-well production, including transient flow, stabilized inflow models, productivity indices, coning and cresting behavior. Although these methods provide insight into the behavior of horizontal wells, only a few of them are considered the pressure drop along the wellbore assuming the infinite conductivity essentially. Horizontal well productivity is limited by the pressure drop within the wellbore, especially when the pressure drop is compared with the reservoir drawdown. A better understanding of the factors affecting the total pressure drop within the wellbore is essential. Knowledge of different pressure drops that effects the horizontal wellbores is crucial in designing successful horizontal wells and optimizing well performance.

In 1990, [1] proposed the first semi analytical model to evaluate the production performance of a horizontal well with the consideration of the wellbore-pressure drop resulting from turbulent flow. Later the study continued by others [2-9] who have presented different coupling models for wellbore flow and reservoir inflow through perforations. However, in certain case studies the pressure drop along a wellbore was studied just by considering only the frictional component. In most circumstances; the pressure drop is studied taking the acceleration into consideration by neglecting the other effects like inflow, mixing etc. Reference [10] in their study revealed that, because of the existence of perforation inflow, acceleration pressure drop is an important factor relative to the frictional component. This significantly will influence the well-flow rates under certain conditions.

With the increase in flow velocity, the momentum influences the pressure drop in addition to the friction pressure drop. This part of the pressure drop has been addressed by several authors in recent years [5, 8, 11, and 12]. Apart from that the perforation holes act like roughness elements which increase the friction factor of the wellbore [13].

Reference [14] performed experimental studies of turbulent 
air flow in a porous circular pipe with uniform air injection through the pipe wall. The fully developed turbulent air flow, at Reynolds numbers of 28,000 to 82,000 , entered the pipe while air was injected uniformly through the wall at different ratios ranging from 0.00246 to 0.0584 of injection velocity to the average velocity at the entrance.

It is quite interesting to note that the characteristics of pipe flow with wall mass transfer are different from those of channel flow or flow past a flat plate. For example, considering the laminar flow case, the local friction factor increases with an increase of wall Reynolds number for pipe flow but decreases for the channel flow [15].

Reference [16] studied flow resistance in a perforated pipe, both with and without flow injection through the pipe wall, by conducting experiments on a pipe of $6^{5 / 6}$ inch outside diameter and $17 \mathrm{ft}$ in length. Reference [11] stated that the total pressure drop along a perforated pipe is made up of wall friction and inflow acceleration and computed the wall friction factor in the same way for a regular, unperforated pipe. Reference [17] studied channel flow with continuous influx into the horizontal channel from an oil-reservoir model. They stated that the pressure gradients increased almost uniformly in the test channel because of the confluence of influx and axial flow, and the resulting pressure drop increases linearly with influx velocity.

A careful set of single-phase experiments in a perforated pipe with radial inflow has been conducted by [18 and 19]. In these experiments, water is used as the working fluid. References [18 and 19] attempt to account for the effects of radial inflow by assuming that the pressure drop in a perforated pipe is the sum of three contributions: the frictional pressure drop, the pressure drop associated with the acceleration of the fluid in the pipe, and finally, a mixing pressure drop. References [18 and 19] showed that most of the pressure drops in the perforated pipe is due to frictional and accelerational effects. However, the mixing pressure drop is significant and its contribution to the pressure drop is often negative. It is, therefore, suggested that the radial inflow lubricates the pipe flow. Although this seems reasonable in the case where the velocity entering through the radial perforations is small compared with the axial velocity in the pipe, they would not expect lubrication to occur when the radial velocity is of the same order of magnitude as the axial velocity. Namely, when radial and axial velocities are of the same magnitude, a jet will penetrate the axial pipe flow resulting in a certain degree of blockage of the pipe. This would lead to an increase of the pressure drop in the pipe.

In this paper, the theoretical that study of the pressure drop in a partially perforated wellbore is presented. That incorporates not only frictional, accelerational pressure drops but also the pressure caused by inflow. The main differences between the theoretical study in this paper with the experiments carried out by [18 and 19] are the diameter of perforations and the perforation density of the pipe. SG has used a perforation diameter of $3 \mathrm{~mm}$ and 158 perforations, where as in this present study, a perforation of $4 \mathrm{~mm}$ diameter and 150 perforations have been used. The objective of this paper is to determine theoretically the various factors that contribute to the total pressure drop in a perforated pipe. In addition to the pressure profiles along a blank section downstream of a perforated section were measured, and new wall-friction-factor correlations for pipe flow with perforation influx were calculated. In line with [18 and 19], it was noticed that the lubrication of the pipe flow occurred when the ratio of the total perforation flow rate to the total flow rate at the pipe outlet was small.

\section{Theoretical Model}

Theoretical analysis was carried out to determine the total pressure drops, frictional, acceleration and additional pressure drops. Fluid flow in a wellbore is considered as shown in Fig. 1 and assumed single-phase flow of an incompressible Newtonian fluid under the isothermal conditions with no heat transfer to and from the fluid to the environment. The test pipe is a partly perforated one and the rest is a plain pipe without perforation. Pipe and perforation geometry for experimental and theoretical study is listed in Table 1. The computational domain taken up in this study is same as that of the dimensions considered in the experimental rigs [18 and 19]. The geometry has been analyzed using three dimensional Computational Fluid Dynamics (CFD). Fig. 2 is the structured computational grids; the mesh consists of 146221 nodes and 542121 elements with five boundary layers. The calculations were carried out with commercial finite volume code ANSYS FLUENT 14 CFX5 using a first scheme and turbulent with $\mathrm{k}$ epsilon model.

Table 1. Geometry of the test pipe.

\begin{tabular}{lll}
\hline Item & Experimental & Theoretical \\
\hline Outer Diameter & $30 \mathrm{~mm}$ & - \\
Inner Diameter & $21.94 \mathrm{~mm}$ & $22 \mathrm{~mm}$ \\
Perfo. Diameter & $3.0 \mathrm{~mm}$ & $4.0 \mathrm{~mm}$ \\
Total perfo. number & 158 & 150 \\
Perfo. phasing & $60^{\circ}$ & $60^{\circ}$ \\
Perfo. density & $12 \mathrm{SPF}$ & $12 \mathrm{SPF}$ \\
\hline
\end{tabular}

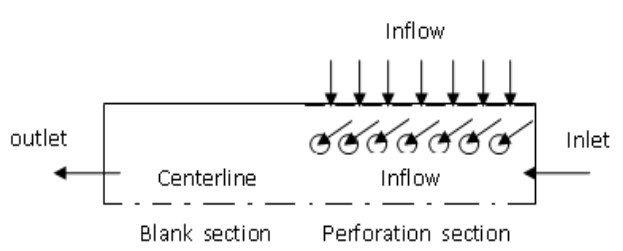

Figure 1. Configuration of partly perforated test pipe (not to scale).

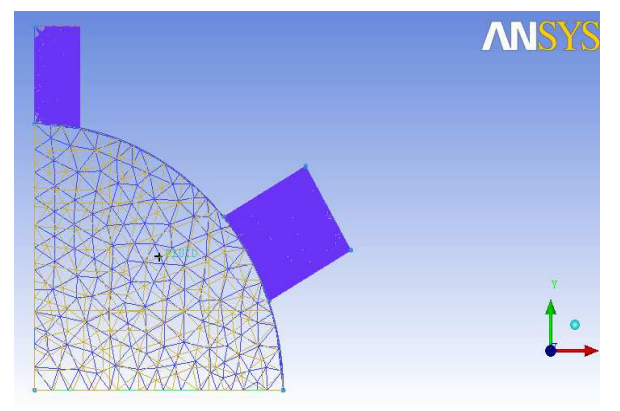

Figure 2.The unstructured mesh for partly perforated pipe. 


\section{Simulation Parameters}

The fluid considered for the simulations is water with constant density of $998.2 \mathrm{~kg} / \mathrm{m}^{3}$ and dynamic viscosity of $0.001 \mathrm{~kg} / \mathrm{m}$ s. Three tests were carried out with Reynolds number of the inlet flow ranging from 28,773 to 90,153 . In each of the tests, flow rate through the perforations was increased from zero to maximum value. The roughness of the test pipe wall was $0.03 \mathrm{~mm}$; the type of the test pipe was PVC.
Test details are summarized in Table 2. Uniform water mass flow is introduced at the inlet of a partially perforated pipe. Two boundary conditions are considered. At the inlet mass flow is taken into consideration both axially and radially where as at the exit outlet pressure is considered as the boundary condition. It is assumed that no-slip boundary conditions occur along the walls. Water enters at a uniform temperature $(\mathrm{T})$ of $25^{\circ} \mathrm{C}$. For the symmetry lines both velocity and pressure is kept constant.

Table 2. Parameters of partly perforated pipe tests.

\begin{tabular}{llll}
\hline Test & Inlet Flow Rate (lit/hr) & Perforation inlet Flow Rate (lit/hr) & Inlet Flow(Re) \\
\hline Test 1 & 5,157 to 5,618 & $0-841$ & 82,756 to 90,153 \\
Test 2 & 3,361 to 3,836 & $0-854$ & 53,935 to 61,557 \\
Test 3 & 1,793 to 2,318 & $0-899$ & 28,773 to 37,198 \\
\hline
\end{tabular}

\section{Pressure Drop in a Pipe with Radial Inflow}

Over a long period of time the pressure drop in a fully developed turbulent pipe flow is being studied by several researchers and investigators. The pressure drop in a straight pipe has been determined in numerous experiments. The total pressure drop in a perforated horizontal wellbore can be divided into a reversible pressure drop and an irreversible pressure drop. The reversible pressure drop is due to the momentum change (flow acceleration) as where more fluid enters the wellbore through perforations. While the irreversible pressure drop is that due to the pipe wall friction, perforation roughness and mixing effects. The following relationship gives the four pressure drop terms that make up the total pressure drop in a perforated horizontal well

$$
\Delta p=\Delta p_{\text {acc. }}+\Delta p_{\text {wall }}+\Delta p_{\text {perfo. }}+\Delta p_{\text {mix }}
$$

The last two terms of Equation (1) combine into one term $\Delta p_{\text {add }}$, which is the pressure drop due to the combined effects of fluid mixing and perforation roughness. Equation (1) can then be rewritten as

$$
\Delta p=\Delta p_{\text {acc. }}+\Delta p_{\text {wall }}+\Delta p_{\text {add }}
$$

Applying the conservation of linear momentum to the control volume in the axial direction for each perforation unit has equal length $\Delta L$, results in the sum of the forces acting on the control volume surfaces towards the downstream direction of the pipe axis

$$
\sum F=m_{\text {out }} u_{\text {out }}-m_{\text {in }} u_{\text {in }}
$$

where the mass flow rate is

$$
m=\rho A u
$$

When radial inflow occurs, the static pressure in the pipe is not uniform, and the velocity profile is not fully developed. In addition to the force contributed by the pressure difference across the control volume and wall shear force, the sum of the forces acting on the control volume surface includes a force due to the combined effects of the irreversible process of fluid mixing and the presence of the perforation hole, including the effect of non-uniformly distribution of static pressure and non-fully developed velocity profile,

$$
\sum F=\left(p_{\text {in }} A-p_{\text {out }} A\right)-\tau_{w}(\pi D \Delta L)-F_{\text {add }}
$$

From the above equations, this can be rearranged to get the following total pressure drop,

$$
p_{\text {in }}-p_{\text {out }}=\rho\left(u_{\text {out }}^{2}-u_{\text {in }}^{2}\right)+\Delta p_{\text {wall }}+\Delta p_{\text {add }} .
$$

Equation (6) indicates that the total pressure drop consists of three different components:

- The pressure drop due to kinematic energy change (acceleration effects). This demonstrates the first term on the right side of Equation (6).

- The frictional pressure drop due to wall friction in a perforation unit, $\Delta p_{\text {wall }}$, is based on the average velocity $u_{\text {out }}$ downstream of the perforation, and can be calculated from the Darcy-Wesibach equation White [20],

$$
\Delta p_{\text {wall }}=\frac{f}{2} \frac{\Delta L}{D} \rho u_{\text {out }}^{2}
$$

When the relative roughness of the pipe is known, an accurate and convenient relationship for the friction factor in the turbulent pipe flow is the Haaland equation

$$
f=\left\{-1.8 \log _{10}\left[\frac{6.9}{\operatorname{Re}}+\left(\frac{\varepsilon}{3.7 D}\right)^{1.11}\right]\right\}^{-2}
$$

For a hydraulically smooth pipe, surface roughness $\mathcal{E}$ should be set to zero.

This equation applies to both laminar and turbulent flow.

The pressure drop due to perforation roughness, $\Delta p_{\text {perfo. }}$, is the extra pressure drop due to the presence of the perforations. It represents the extra friction caused by the perforations 
acting as roughness elements in the pipe wall. The pressure drop due to perforation roughness is most important when there is no flow through the perforations. It has been shown that the magnitude of the pressure drop due to perforation roughness depends on the pipe-perforation geometry and the perforation density [21].

The pressure drop due to mixing effects, $\Delta p_{\text {mix. }}$, is an irreversible pressure drop which cannot be further classified. This pressure effect arises from the complex interaction between perforation flow and wellbore flow, which causes disturbances in the boundary layer and hence affects the pressure drop. The irreversible pressure drop due to mixing needs to be determined by experiments [21].

\section{New Wall-Friction-Factor Correlations [10]}

Mass transfer through the pipe wall affects the wall-friction shear. The influence of either inflow or outflow depends on the regime present on the wellbore. The inflow (production well) increases the wall friction for laminar flow while decreasing it for turbulent flow. In contrast, outflow (injection well) decreases the wall friction for laminar flow while increasing it for turbulent flow. In other words, the wall friction is different from that of pipe flow with no inflow or outflow. Therefore, friction -factor correlations for pipe flow without inflow or outflow cannot be used for wellbore flow with both axial flow in the pipe and inflow or outflow through perforations [10].

A new correlation for the local wall friction factor for turbulent flow has been developed in [10] using Olson and Eckert's experimental data[14] for turbulent air flow in a porous pipe with uniform air injection through the pipe wall. The new correlation is of the form [10]

$$
f=f_{o}\left[1-29.03\left(\frac{\mathrm{Re}_{w}}{\mathrm{Re}}\right)^{0.8003}\right]
$$

It was found that the ratio between the local friction factor and the no-wall flow friction factor does not depend on the wall Reynolds number to axial Reynolds number ratio; instead, it depends only on the wall Reynolds number. Therefore, a new correlation for the local friction factor was developed [10]

$$
f=f_{o}\left[1-0.0153 \operatorname{Re}_{w}^{0.3978}\right]
$$

Eq. 10 is a satisfactory correlation for local wall friction factor for single-phase turbulent wellbore flow [10].

\section{Results and Discussions}

\subsection{Pressure Drops in Perforated Section}

In this paper, theoretically were carried out on the pipe that was simulated with the experimental pipe. Three tests with different pipe flow rate were carried out for the perforated pipe.
The analytical results were examined in terms of the total pressure drop, as shown in Fig. 3. The individual tests had an average outlet flow Reynolds number in the range of 37,460 to 108,940 . The total flow rate ratio (q) is the total perforation flow rate divided by the total flow rate at pipe outlet.

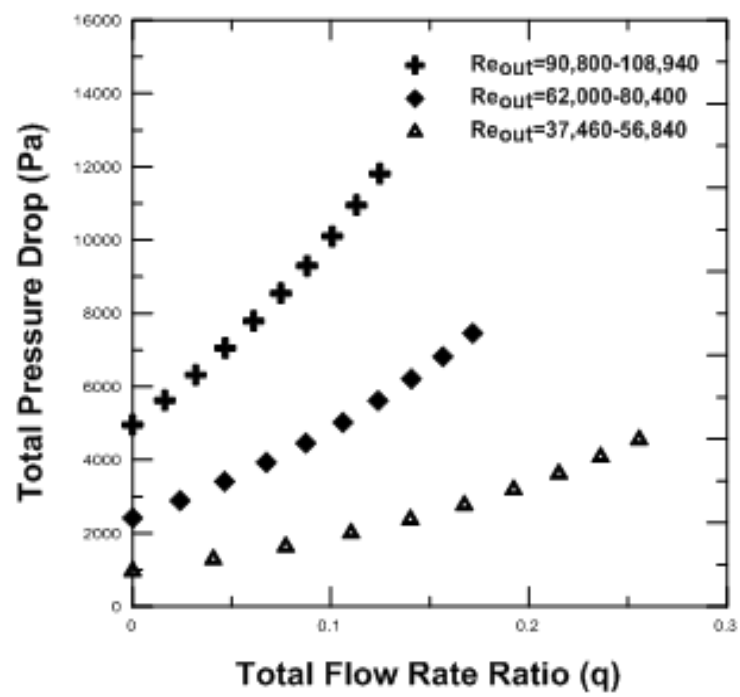

Figure 3.Total pressure drop across perforated section.

The total pressure drop was found to be higher for higher Reynolds numbers. This effect was caused by the larger wall frictional pressure drop under higher flow velocity. As the rate of flow through the perforations increases, the flow rate ratio increases and the total pressure drop increases. The main reason is that a higher flow rate through the perforations giving a larger acceleration pressure drop. In addition, it was found that greater wall friction was due to larger average flow velocity in the pipe, which was caused by inflow through the perforations and increased mixing effect.

The pressure drop due to momentum change (acceleration pressure drop) was calculated from Equation (6) (the first term of the right side). It was noticed that the values of acceleration pressure drop for partly perforated wellbore were higher than the values of the frictional pressure drop.

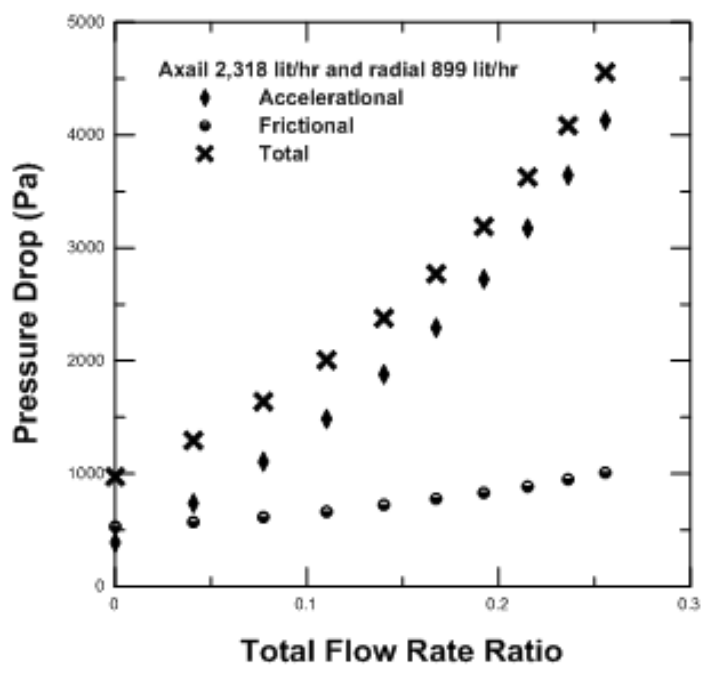

Figure 4. Pressure drops across perforated section for Test3. 
The acceleration pressure drop is also very important and cannot be neglected from the inference of Fig. 4. Reference [10] explained that for partly perforated wellbore, the ratio of the acceleration pressure drop to the frictional pressure drop $R_{a f}$ is higher and changes from 1.27 to 0.71 . The cumulative acceleration pressure drop from starting point of the perforations is more or less the same as the cumulative frictional pressure drop. It is shown that the acceleration pressure drop may or may not be important compared to the frictional component depending on the specific pipe geometry, fluid properties and flow conditions.

The increase in total flow rate ratio q leads to an increase in the pressure drop and further this leads to increase in the axial pressure drop.

Fig. 5 represents the total pressure drop and acceleration pressure drop for three tests. For tests 1 and 2 the acceleration pressure drop is approximated to zero value but test 3 has values greater than values of test 1 and test 2 at zero value of total flow rate ratio. The acceleration pressure drop contributes to the important part of the total pressure drop for all the three tests. The acceleration component contributes in the range of $36.6 \%$ to $99.9 \%$ for test 1 , from $13 \%$ to $99.9 \%$ for test 2 , and for test 3 from $9.3 \%$ to $59.8 \%$.

From Equation (6), subtracting the acceleration pressure drop from the total pressure drop $\Delta p-\rho\left(u_{\text {out }}^{2}-u_{\text {in }}^{2}\right)$, the results are equal to the summation of pressure drop due to wall friction and additional pressure drop. The additional pressure includes pressure drop due to perforation roughness and mixing flow $\Delta p_{\text {add. }}$.

Fig. 6 depicts the behavior of the remaining pressure drop. The remaining pressure drop decreases with increase in total flow rate ratio. As the rate of flow through the perforations increases, the flow rate ratio increases correspondingly and the total pressure drop increases. It is due to the larger acceleration pressure drop for higher flow rate through the perforations. The remaining pressure drop decreases with the increasing value of the total flow rate ratio. Due to this, the acceleration pressure drop increases with the increase in the total flow rate ratio.

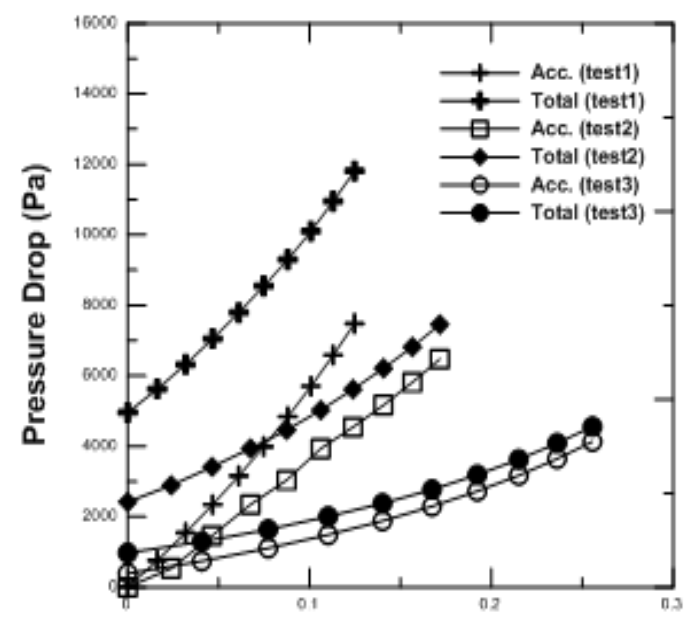

Total Flow Rate Ratio

Figure 5. Total and acceleration pressure drops for the three tests.

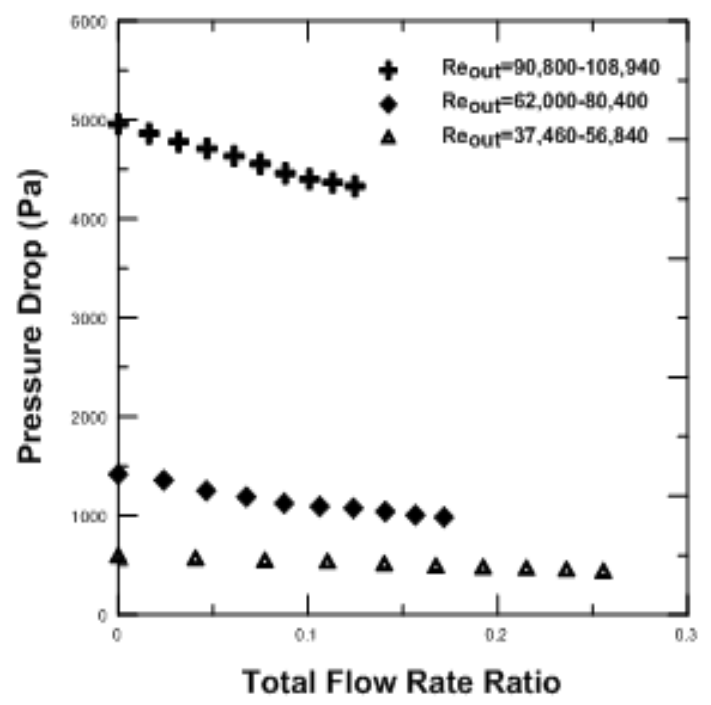

Figure 6. Pressure drop due to wall friction, perforation roughness, and flow mixing.

\subsection{Additional Pressure Drop in Perforated Section}

The additional pressure drop is the resultant of the mixing effect and the perforation roughness. The additional pressure drop is obtained by subtracting the frictional pressure drop from the remaining pressure drop. The wall friction pressure drop of a perforated section was calculated using Equation (7) for a unit perforation. The friction factors were calculated from Equation (8) for perforated pipe using $0.03 \mathrm{~mm}$. The roughness of the pipe was taken into consideration because the pipe was made of PVC.

The wall friction pressure drop for uniformly perforated section was calculated Equation (11). Using Equation (8), the friction factor was calculated. The friction factor can also be found from Equation (9) because it depends on the ratio of $\left(\frac{\mathrm{Re}_{w}}{\mathrm{Re}}\right)$ or from Equation (10) because it depends only on the wall Reynolds number [10]

$$
\Delta p_{\text {wall }}=\sum_{i=1}^{N-1}\left(f_{i} \frac{\Delta L_{i}}{D} \frac{\rho u_{i}^{2}}{2}\right)
$$

where $\mathrm{N}$ is the total number of perforations and $\mathrm{D}$ is the pipe diameter. The value of $\Delta \mathrm{L}_{\mathrm{i}}$ is the distance in the axial direction of the pipe between two adjacent perforations minus the equivalent length that is occupied by a perforation. For a uniformly perforated pipe, $\Delta \mathrm{L}_{\mathrm{i}}$ is a constant, calculated as

$$
\Delta L_{i}=\frac{L}{N-1}-\frac{d^{2}}{4 D}
$$

where $d$ is the perforation diameter and $L$ the total length between the first and the last perforation of the perforated section.

Fig. 7 represents the additional pressure drop with total flow rate ratio for all the tests. The additional pressure drop 
decreases as the flow rate ratio increases. When the flow rate ratio is zero, the additional pressure drop is caused by perforation roughness only. When the total flow rate ratio reaches a certain limit, which is about 0.045 for test 1 and 2, the pressure drop caused by the perforation is eliminated by the smoothing effect. Beyond this limit of the flow rate ratio, the additional pressure drop has a negative value, which means that the pressure drop due to wall friction is reduced by the smoothing effect. For test 3 , we show that all the additional pressure drop values are negative because of increases in radial flow, except for the value at zero flow rate ratio.

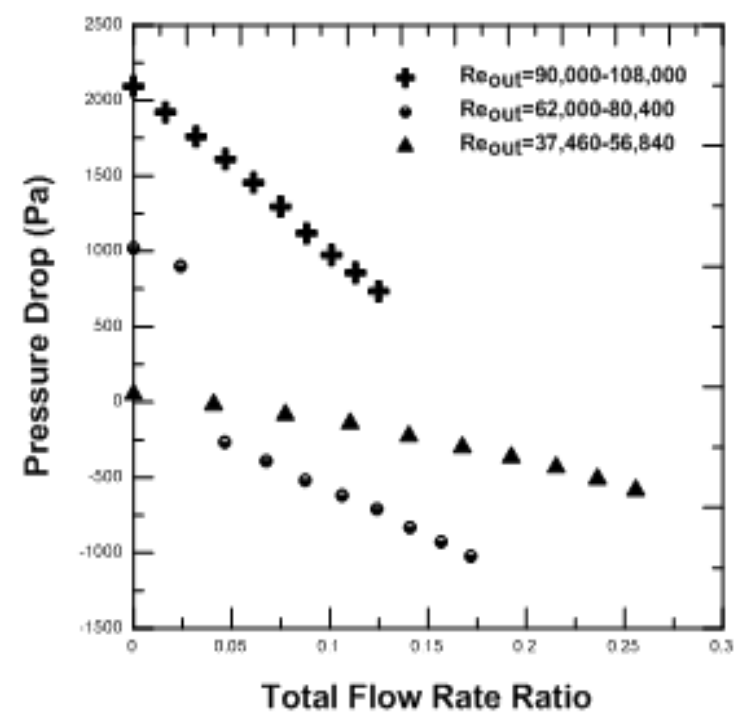

Figure 7. Additional pressure drop.

\subsection{Total Pressure Drop in Blank Section}

Total pressure drop was calculated for plain pipe section without perforations with flow rate ratio as shown in Fig. 8. The perforated section is followed by the plain section. The values of the total pressure drop in the plain section are lower than the values of total pressure drop for the perforated section because the pressure drop in the plain section of the pipe is mainly due to the pipe wall friction.

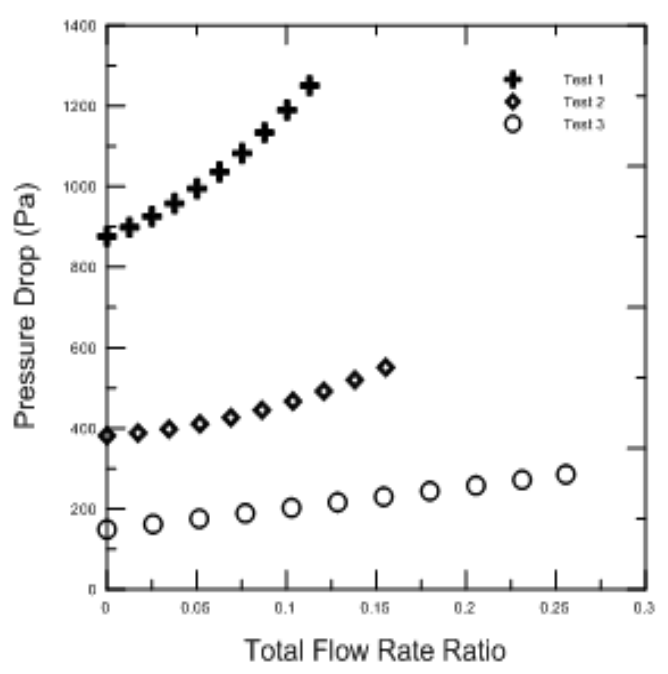

Figure 8. Total pressure drop for blank section.

\subsection{Pressure Drop Coefficients}

Pressure drop in a perforated pipe is the function of the flow rate in the pipe. A pressure drop coefficient is defined as the pressure drop across the perforated section divided by the kinetic energy at the outlet of the pipe.

$$
\mathrm{K}=\frac{\Delta \mathrm{p}}{0.5 * \rho * \mathrm{u}_{\mathrm{out}}^{2}}
$$

where $u_{\text {out }}$ is the average flow velocity at the outlet of the test pipe.

The pressure drop coefficients were calculated for total and additional pressure drops for perforated section for all the three tests. The data points for each test follow a straight line as shown in Fig. 9. Data points of the three tests for total pressure drop followed parallely and closely except for some points of those tests conducted with Reynolds number range from 62,000 to 80,400 and from 37,460 to 56,840 . The pressure drop coefficients increase linearly with increasing total flow rate ratio.

Data points of different tests for additional pressure are shown in Fig. 10. Here also the graphical lines follow closely and parallel at low Reynolds number except for the tests conducted with Reynolds number ranges from 90,800 to 108,940 ( due to high effect of pressure drop with high Re). So increase Re range increase the pressure drop, increase of mass flow with high Re and low kinetic energy at the perforated section. The data points decrease with increasing total flow rate ratio as shown in Fig 6.

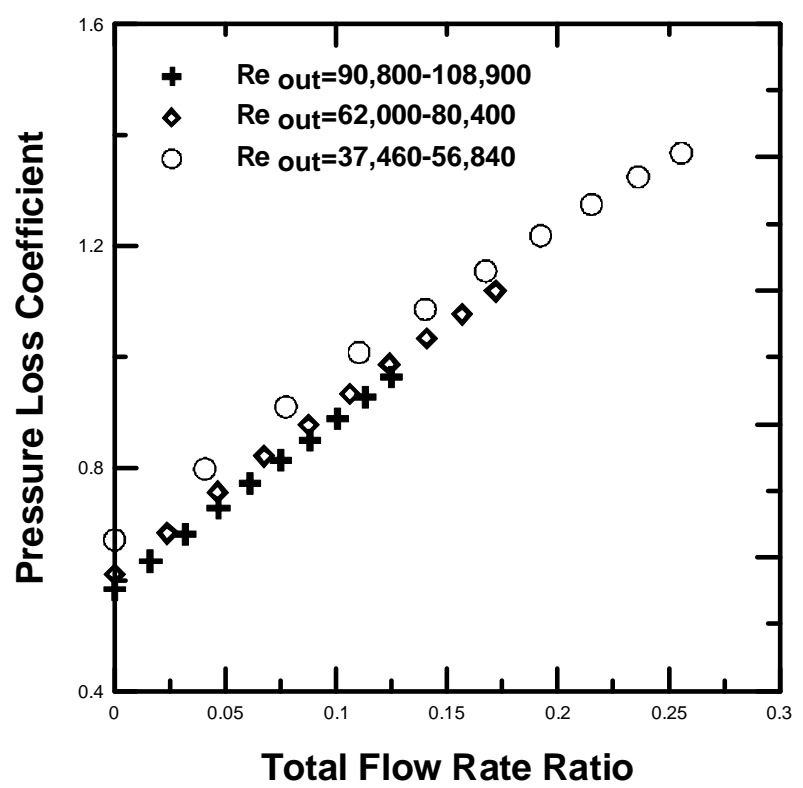

Figure 9. Pressure drop coefficient for total pressure drop. 


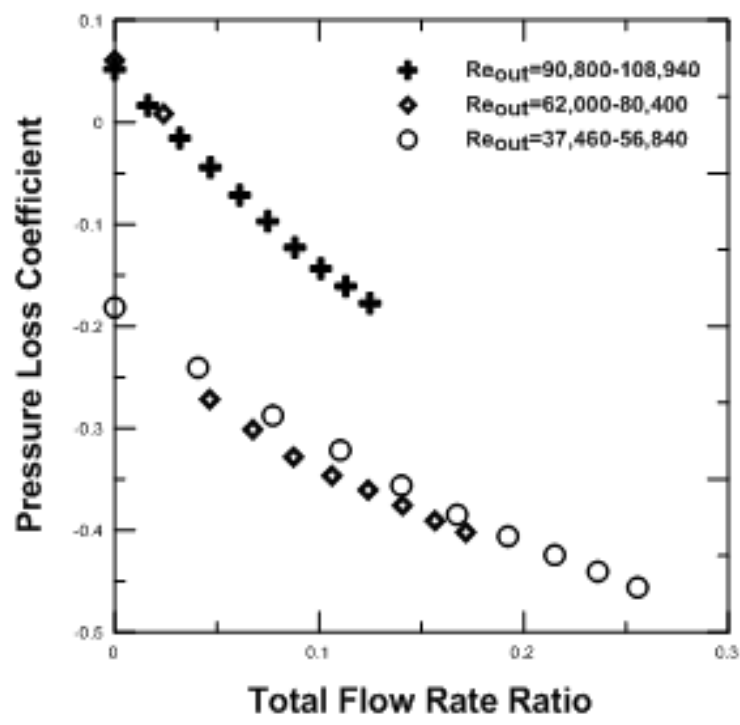

Figure 10. Pressure drop coefficient for additional pressure drop.

Fig 11 depicts the Numerical Simulation results in this paper and the experimental results conducted in tests[21] were used for the comparison of the total pressure drop in the perforated section between the first perforation and the last perforation.

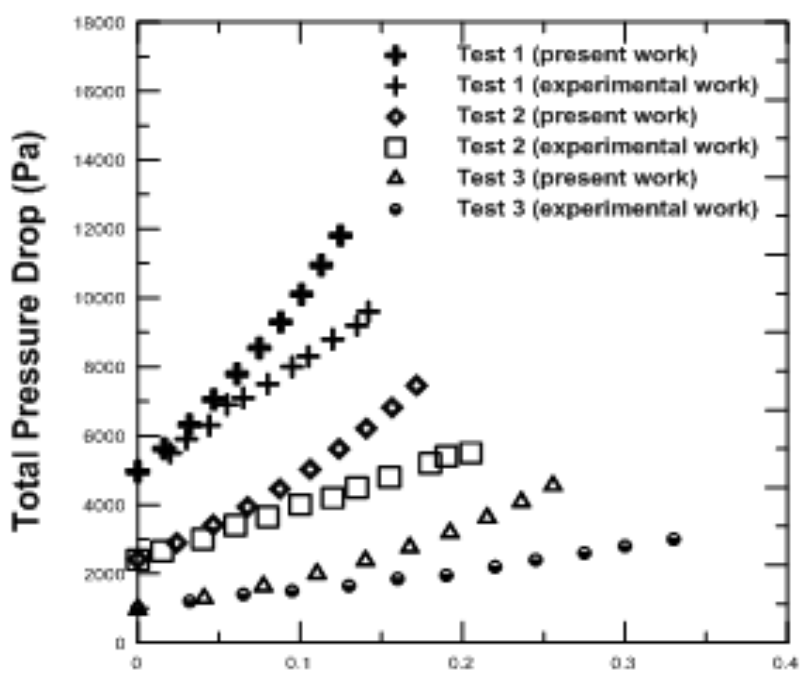

Total Flow Rate Ratio

Figure 11. Comparison of numerical and experimental data[21].

It further shows that there is a difference for all the tests especially when the total flow rate increases and the curves are diverging from the experimental tests. The reason for this is at the range of values of the Reynolds numbers is different and higher than from the experimental tests.

\subsection{Friction Factor}

Friction factor is a dimensionless parameter extensively used in pipe flows to express the pressure drop due to frictional effect. Fig.12 represents the new correlation prediction by Equation (9) for local friction factor ratio $f / f_{o}$ with injection Reynolds number $\mathrm{Re}_{w}$. It was observed that the local friction factor ratio for a perforated pipe with fluid injection for all the tests conducted above decreases when the radial flow increases. For test 1, the values of the local friction factor ratio is higher than tests 2 and 3. From Equation (9), it was observed that with the increase in the value of the radial flow, the local friction factor ratio decreased.

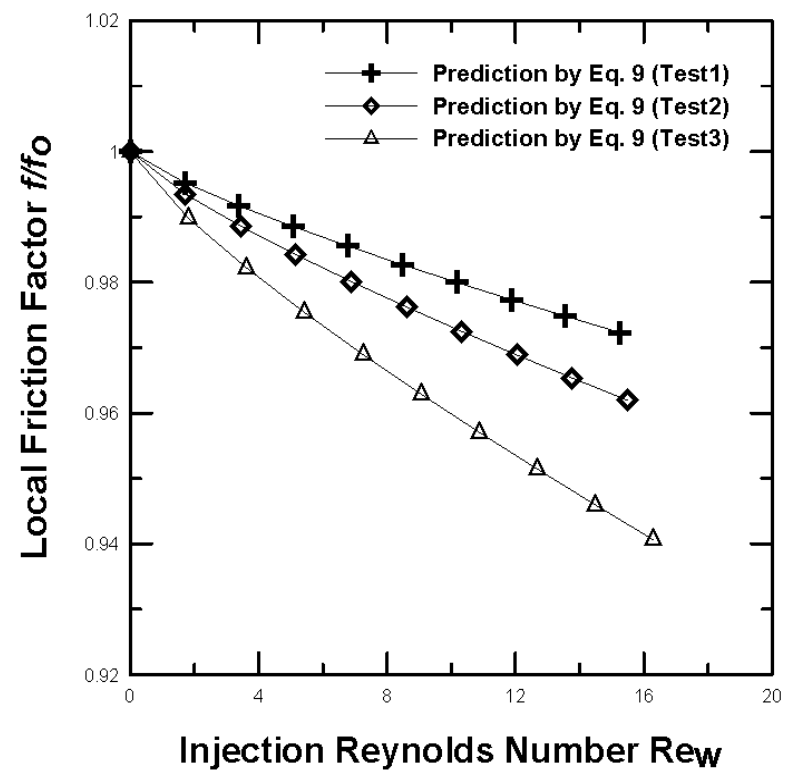

Figure 12. Wall friction factor correlation for turbulent pipe flow with inflow.

Fig. 13 represents the new correlation prediction by Equation (10) for local friction factor ratio $f / f_{o}$ with injection Reynolds number $\mathrm{Re}_{w}$. It was observed that all the data points of the three tests pertaining to the friction factor for a perforated pipe with fluid injection were close to each other because the results depended on $\mathrm{Re}_{w}$ only.

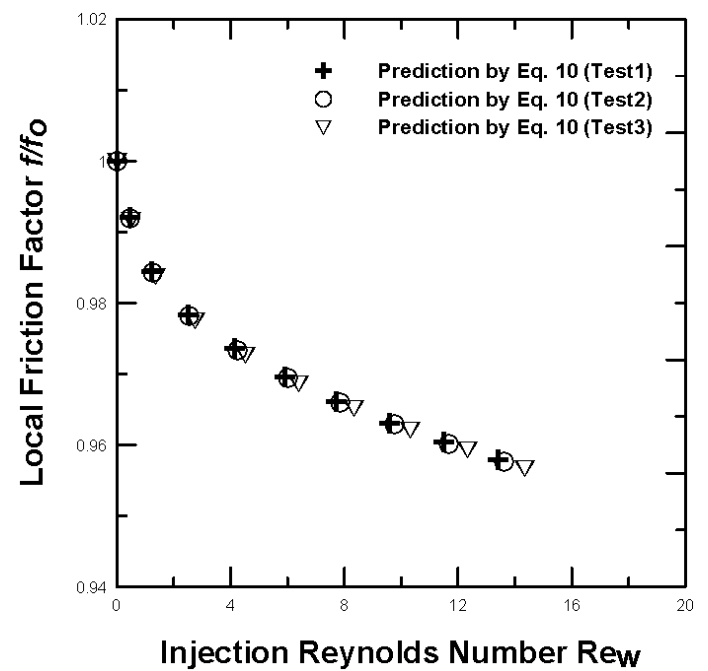

Figure 13. Wall friction factor correlation for turbulent pipe flow with inflow.

\section{Conclusions}

Numerical simulations have been carried out on the flow in a partly perforated pipe with inflow through perforations. The geometry of the pipe used was similar to the pipe used in the 
experimental tests [18, 19 and 21]. The total pressure drop in a horizontal wellbore is the sum of the pressure drops due to momentum change (acceleration), wall friction, perforation roughness, and fluid mixing. The acceleration pressure drop cannot be ignored compared with the frictional pressure drop. The total pressure drop for perforated section was larger than the total pressure drop in the plain section without perforations. The additional pressure drop caused by the perforation roughness was eliminated by the smoothing effect once the flow rate ratio reached a certain limit. It was observed that the local friction factor ratio for a perforated pipe with fluid injection decreased with increase of the radial flow.

\section{References}

[1] Dikken, B.J., "Pressure Drop in Horizontal Wells and its Effect on Production Performance," JPT (November 1990) 1426.

[2] Islam, M.R. and Chakma, A., "Comprehensive Physical and Numerical Modeling of a Horizontal Well," paper SPE 20627 presented at the 1990 SPE Annual Technical Conference and Exhibition, New Orleans, 23-26 September.

[3] Folefac, A.N. et al. "Effect of pressure Along Horizontal Wellbore on Well Performance, Aberdeen, 3-6 September.

[4] Ozkan, E., Sarica, C., and Haciislamoglu, M.: "Effect of Conductivity on Horizontal Well Pressure Behavior," paper SPE 24683 presented at the 1992 SPE Annual Technical Conference and Exhibition, Washington, Dc, 4-7 October.

[5] Ihara, M. and Shimizu, N., "Effect of Acceleration Pressure Drop in a Horizontal Wellbore," paper SPE 26519 presented at the 1993 SPE Annual Technical Conference and Exhibition, Houston, 3-6 October.

[6] Seines, K. et al., "Considering Wellbore Friction Effects in Planning Horizontal Wells,” JPT (October 1993) 994.

[7] Landman, M.J., "Analytical Modeling of Selectivity Perforated Horizontal Wells," J. Petroleum Science and Engineering (1994) $10,179$.

[8] Sarica, C. et al., "Influence of Wellbore Hydraulics on Pressure Behavior and Productivity of Horizontal Wells," paper SPE 28486 presented at the 1994 SPE Annual Technical Conference and Exhibition, New Orleans, 25-28 September.
[9] Novy, R.A., "Pressure Drops in Horizontal Wells: When Can They be Ignored?” SPERE (1995) 29.

[10] Ouyang, L.B. et al., "A Single-Phase Wellbore-Flow Model for Horizontal, Vertical, and Slanted Wells," SPE Journal 3(2), 1998, pp. 124-133.

[11] Asheim, H. et al., "A Flow Resistance Correlation for Completed Wellbore," J. Petrol. Sci. Eng., 1992, 8 (2), pp. 97-104.

[12] Marett, B.P., Landman, M.J., "Optimal Perforation Design for Horizontal Wells in Reservoir with Boundaries," paper SPE 25366 presented at the 1993 SPE Asia Pacific Oil and Gas Conference and Exhibition, Singapore, February 8-10.

[13] Su, Z., Gudmundsson, J.S., "Friction Factor of Perforation Roughness in Pipes," SPE 26521 presented at the 1993 SPE $68^{\text {th }}$ Annual Technical Conference and Exhibition, Houston, TX, USA, October 3-6.

[14] Olson, R.M. and Eckert, E.R.G., "Experimental Studies of Turbulent Flow in a Porous Circular Tube with Uniform Fluid Injection through the Tube Wall," J. Applied Mechanics (1966) 33 , No. $1,7$.

[15] Rabithby, G., "Laminar Heat Transfer in the Thermal Entrance Region of Circular Tubes and Two-Dimensional Rectangular Ducts with Wall Suction and Injection," Intl. J. Heat and Mass Transfer (1971) 14, No. 2, 223.

[16] Kloster, J., "Experimental Research on Flow Resistance in Perforated Pipe," Master thesis, Norwegian Int. of Technology, Trondheim, Norway (1990).

[17] Ihara, M. et al., "Flow in Horizontal Wellbores with Influx through Porous Walls," paper SPE 28485 presented at the 1994 SPE Annual Technical Conference and Exhibition, New Orleans, 25-28 September.

[18] Su, Z. and Gudmundsson, J.S., "Pressure Drop in Perforated Pipes," PROFIT Projected Summary Reports, Norwegian Petroleum Directorate, Stavanger (1995).

[19] Su, Z. and Gudmundsson, J.S., "Pressure Drop in Perforated Pipes," report, Department of Petroleum Engineering and Applied Geophysics, U. Trondheim, Norway (1995).

[20] White, F.M., "Fluid Mechanics,” McGraw-Hill, Inc. 1986.

[21] Su, Z. and Gudmundsson, J.S.: "Perforation Inflow Reduces Frictional Pressure Loss in Horizontal Wellbores,” J. Petrol. Sci. Eng., 1998, 19, pp. 223-232. 